\title{
Acute ethanol treatment upregulates th1, th2, and hdc in larval zebrafish in stable networks
}

\author{
Henri A. J. Puttonen, Maria Sundvik, Stanislav Rozov, Yu-Chia Chen and Pertti Panula* \\ Neuroscience Center and Institute of Biomedicine/Anatomy, University of Helsinki, Helsinki, Finland
}

\section{Edited by:}

Gonzalo G. De Polavieja, Instituto

Cajal, CSIC, Spain

Reviewed by:

Gonzalo G. De Polavieja, Instituto Cajal, CSIC, Spain

Caroline H. Brennan, Queen Mary

University of London, UK

*Correspondence:

Pertti Panula, Neuroscience Center and Institute of Biomedicum,

University of Helsinki, PO Box 63 ,

Haartmaninkatu 8, FIN-00014

Helsinki, Finland

e-mail: pertti.panula@helsinki.fi
Earlier studies in zebrafish have revealed that acutely given ethanol has a stimulatory effect on locomotion in fish larvae but the mechanism of this effect has not been revealed. We studied the effects of ethanol concentrations between 0.75 and $3.00 \%$ on 7-day-old larval zebrafish (Danio rerio) of the Turku strain. At $0.75-3 \%$ concentrations ethanol increased swimming speed during the first minute. At $3 \%$ the swimming speed decreased rapidly after the first minute, whereas at 0.75 and $1.5 \%$ a prolonged increase in swimming speed was seen. At the highest ethanol concentration dopamine levels decreased significantly after a 10-min treatment. We found that ethanol upregulates key genes involved in the biosynthesis of histamine ( $h d c)$ and dopamine (th 1 and th2) following a short 10-min ethanol treatment, measured by qPCR. Using in situ hybridization and immunohistochemistry, we further discovered that the morphology of the histaminergic and dopaminergic neurons and networks in the larval zebrafish brain was unaffected by both the 10-min and a longer 30-min treatment. The results suggest that acute ethanol rapidly decreases dopamine levels, and activates both forms or th to replenish the dopamine stores within $30 \mathrm{~min}$. The dynamic changes in histaminergic and dopaminergic system enzymes occurred in the same cells which normally express the transcripts. As both dopamine and histamine are known to be involved in the behavioral effects of ethanol and locomotor stimulation, these results suggest that rapid adaptations of these networks are associated with altered locomotor activity.

Keywords: zebrafish, ethanol, tyrosine hydroxylase, histidine decarboxylase, behavior

\section{INTRODUCTION}

Ethanol is the most widely used recreational drug. Ethanol abuse has been associated with many diseases of the brain and hazardous behavior, and due to its increasing prevalence, it is crucial to understand the effects of ethanol on the brain in detail. The dopaminergic system is one of the many targets of ethanol, and it has been widely studied in several models. Earlier studies have shown that the dopaminergic neurons in the VTA in rats are activated by ethanol administration (Gessa et al., 1985). Furthermore, studies have shown that the extracellular dopamine concentration in target areas of these neurons, mainly the nucleus accumbens, increases after ethanol exposure both in rats (Di Chiara and Imperato, 1986; Yoshimoto et al., 1992) and in mice (Tang et al., 2003). The activation of this mesolimbic dopamine pathway has been linked to mechanisms of substance addiction and reinforcement (Di Chiara and Imperato, 1988) and the increase in locomotor activity observed upon administration of ethanol in rodents (Cohen et al., 1997). Although the changes in dopamine levels are well known, the acute effect of ethanol on the expression of tyrosine hydroxylase $(\mathrm{TH})$, the ratelimiting enzyme in dopamine synthesis, is not well-understood, with some evidence reporting an increase in th mRNA levels (Oliva et al., 2008). A chronic treatment with ethanol has been shown to increase th mRNA levels in the rat brain (Lee et al., 2005; Navarrete et al., 2013), but there is also evidence suggesting that $\mathrm{TH}$ protein levels decrease after chronic ethanol treatment (Kashem et al., 2012).

In mammals, the forebrain nucleus accumbens has been considered a critical region for transforming motivational and cognitive inputs to action since the initial concept was presented (Mogenson et al., 1980). Dopamine release in this region is needed for the locomotor activation induced by stimulatory drugs in mammals (Kelly et al., 1975; Farrar et al., 2010). The related functional neural circuits have not been analyzed in zebrafish, although both low concentrations of alcohol and d-amphetamine in swimming water significantly increase locomotor activity in larval zebrafish (Irons et al., 2010). Since the zebrafish do not possess dopaminergic neurons in the mesencephalon, significant efforts have been devoted to identifying the origin of subpallial dopaminergic inputs which might correspond to the mammalian striatonigral dopaminergic system necessary for initiation of voluntary movements. Both in adult zebrafish (Kaslin and Panula, 2001) and early larval zebrafish (McLean and Fetcho, 2004), local $\mathrm{TH}$ immunoreactive cells give rise to local projections. The $\mathrm{TH}$-immunoreactive neurons in the posterior tuberculum also give rise to ascending projections toward the subpallium (Kaslin and Panula, 2001; Rink and Wullimann, 2001). A detailed analysis of individual neurons of all dopaminergic clusters using th:rasEGFP transgenic fish suggests that at an early stage ( $4 \mathrm{dpf}$ ) only very few dopaminergic neurons in the posterior tuberculum 
send ascending projections, whereas most of the fibers are of intrinsic subpallial origin (Tay et al., 2011). The current concept is that both intrinsic and ascending projections contribute to the dopaminergic inputs to subpallium. Many diencephalic dopaminergic cell clusters give rise to extensive descending projections to the rhombencephalon and spinal cord in both larval and adult zebrafish (Kaslin and Panula, 2001; McLean and Fetcho, 2004; Tay et al., 2011). Thus, dopamine can potentially regulate movement on all levels from telencephalon to spinal cord, where dopaminergic fibers are concentrated in the ventral horn (McLean and Fetcho, 2004).

Although less studied, the histaminergic system is known to be affected by ethanol. Acutely administered ethanol increases histamine content in rodents (Subramanian et al., 1978; Rawat, 1980), with one study reporting a decrease with higher ethanol doses (Papanicolaou and Fennessy, 1980). Histamine has also been associated with ethanol tolerance in rats, with ethanol sensitive alcohol non-tolerant rats showing lower levels of brain histamine than ethanol insensitive alcohol tolerant rats and decreased ethanol tolerance in alcohol tolerant rats upon pharmacological inhibition of histamine synthesis (Lintunen et al., 2002). Recent studies have also shown that knocking out the histamine synthesizing enzyme histidine decarboxylase (HDC) in mice inhibits the increase of locomotor activity caused by ethanol (Nuutinen et al., 2010), and mice treated with histamine H3 receptor antagonists or those lacking $\mathrm{H} 3$ receptor do not show ethanol-induced conditioned place preference (Nuutinen et al., 2011). In the light of these new discoveries, the histaminergic system can be considered a major area of interest in ethanol research.

A vast majority of reported ethanol studies have utilized different rodent models. Lately, the zebrafish has been established as an alternative model to study the effects of ethanol. Behavioral analysis methods suitable for ethanol research have been established both for adult (Gerlai et al., 2000; Kily et al., 2008; Mathur et al., 2011) and larval zebrafish (Lockwood et al., 2004). There are also several advantages to using zebrafish, including the possibility to visualize whole neurotransmitter networks in vivo in larval stages of the fish, as has been shown in several earlier studies (Chen et al., 2009; Sallinen et al., 2009b). An acute exposure of adult zebrafish to ethanol increases brain dopamine levels in a dose-dependent manner (Chatterjee and Gerlai, 2009), but the kinetics of these changes which potentially modify the functions of important circuits are not known. Furthermore, the regulation of the rate-limiting dopamine synthesizing enzyme $\mathrm{TH}$ has not been studied. We hypothesized that the increased dopamine levels should be associated with essential changes in synthesizing enzymes TH, and aimed at identifying which of the two TH forms is activated. We also hypothesized that synthesis of histamine, another amine involved in increased vigilance and motor activity, could undergo essential changes following ethanol exposure. In this study, we used 7-day-old larval zebrafish to study the effect of several acute short-term ethanol treatments on swimming behavior. In addition, we associated the changes observed to possible functional or anatomical alterations in key markers of the histaminergic and dopaminergic circuits using confocal analysis on whole neuronal networks and in situ hybridization.

\section{MATERIALS AND METHODS EXPERIMENTAL ANIMALS}

Larval zebrafish of a wild-type Turku strain were used in all experiments. This strain has been maintained in the laboratory for more than a decade, and has been used in several earlier publications (Chen et al., 2009; Sallinen et al., 2009a). Fish were bred and maintained according to Westerfield (Westerfield, 2000). A permit for the experiments was obtained from the Office of the Regional Government of Southern Finland. All batches of embryos were obtained from several parent fish in order to minimize the chance of a rare genotype affecting the results.

\section{ETHANOL TREATMENT FOR HISTOLOGIC AND OUANTITATIVE METHODS}

Ten to twenty 7dpf (days post-fertilization) larval zebrafish were transferred to a six-well plate in $1 \times \mathrm{E} 3$ (zebrafish embryonic medium; $5.00 \mathrm{mM} \mathrm{NaCl}, 0.44 \mathrm{mM} \mathrm{CaCl}_{2}, 0.33 \mathrm{mM} \mathrm{MgSO}_{4}$ and $0.17 \mathrm{mM} \mathrm{KCl})$. The $1 \times \mathrm{E} 3$ was then replaced with $1 \times \mathrm{E} 3$ containing ethanol. Four different ethanol concentrations were used: $0.00 \%$ (control), $0.75,1.50$, and $3.00 \%(\mathrm{v} / \mathrm{v})$. These concentrations were chosen based on previous studies with zebrafish and ethanol (Lockwood et al., 2004; Chatterjee and Gerlai, 2009). The treatment durations used were 10 and $30 \mathrm{~min}$. Following the ethanol treatment, the larvae were quickly collected into $1.5 \mathrm{ml}$ microcentrifuge tubes and sacrificed on ice.

\section{BEHAVIORAL ANALYSIS}

The locomotor activity of 7dpf larvae after ethanol exposure was observed during a 10-min time period. Forty eight larvae were tracked at a time on a 48-well plate, as described previously (Peitsaro et al., 2007). The larvae were divided into four treatment groups of 12 larvae. Ethanol solutions used were the same as those described above. The solution was administered carefully into the wells immediately prior to the tracking in order to assess the acute locomotor effect of ethanol exposure. When analysing total distance moved, fish that did not swim at all during the trial were considered outliers and excluded from the analysis. The amount of larvae excluded from each of the groups due to zero movement was as follows: control: 1 out of $48,0.75 \%$ : 5 out of $61,1.50 \%: 7$ out of 68 and $3.00 \%: 2$ out of 55 . The values were transformed using $\log _{10}$ transformation, after which the values conformed better to a normal distribution and were analysed using 1-way ANOVA followed by Tukey's post-hoc test. Other variables were analysed without the use of any transformations using the same statistical tests. Additionally, total distance moved was also analysed with 1-min intervals, with statistical analysis done using Two-Way repeated measures (RM) ANOVA followed by Bonferroni's post hoc test after performing a $\log _{10}$ transformation as described above. Fish that did not move at all during one or more intervals were assigned the value $10^{-4}$ for total swimming distance during those time points to enable use of a $\log _{10}$ transformation.

\section{QUANTITATIVE PCR}

Groups of 15 larvae were treated with ethanol for $10 \mathrm{~min}$ as described above. Total RNA was isolated using the RNeasy Mini Kit (Qiagen, Hilden, Germany), followed by cDNA synthesis 
using the SuperScriptIII kit (Invitrogen). The SmartCyclerII ${ }^{\circledR}$ cycling platform was used for $\mathrm{qPCR}$. The reaction mix consisted of SYBR Green premix (Takara, Madison, WI, USA), primers and cDNA template. The primer sequences have been described earlier (Sallinen et al., 2010; Pavlidis et al., 2011). Quantification was done by $\mathrm{Ct}$ value comparison, using the $\mathrm{Ct}$ value of $\beta$-actin as an internal standard (Livak and Schmittgen, 2001). Statistical analysis was done using One-Way ANOVA followed by Tukey's post-hoc test.

\section{TYROSINE HYDROXYLASE 1 AND 2, AND HISTIDINE DECARBOXYLASE WHOLE-MOUNT in situ HYBRIDIZATION}

Antisense digoxigenin (DIG)-labeled RNA probes were synthesized using the DIG RNA labeling kit (Roche Diagnostics, Germany). The clones used have been described previously (Chen et al., 2009; Sundvik et al., 2011). Larvae samples for wholemount in situ hybridization (WISH) were collected after ethanol treatment and fixed in 4\% PFA (paraformaldehyde) in PBS overnight. WISH was carried out according to the Thisse lab protocol with minor modifications (Thisse and Thisse, 2008). The heads were dissected after fixation in order to expose the brain. All hybridization steps were done at $67^{\circ} \mathrm{C}$. The probes were detected using NBT (nitro blue tetrazolium, Roche diagnostics $\mathrm{GmBH}$, Mannheim, Germany)/BCIP (5-bromo 4-chloro 3-indolyl phosphate, Roche diagnostics GmBH, Mannheim, Germany).

\section{TYROSINE HYDROXYLASE AND HISTAMINE IMMUNOHISTOCHEMISTRY}

Larvae were collected after ethanol treatment and fixed overnight in 4\% EDAC (1-ethyl-3,3 (dimethyl-aminopropyl) carbodiimide) and $0.1 \%$ PFA in PBS for tyrosine hydroxylase (TH1) and histamine double staining. Samples used only for TH1 staining were fixed in 4\% PFA in PBS. The detailed protocol for immunohistochemistry has been described earlier (Sallinen et al., 2009b). For primary antibodies, monoclonal mouse anti-TH1 antibody (Diasorin/Immunostar, lot no. 22941) diluted 1:1000 and polyclonal rabbit anti-Histamine serum [rabbit anti-histamine 19C (Panula et al., 1990)] diluted 1:10000 were used. For detection, the samples were incubated with Alexa-conjugated antibodies (Alexa Anti-Mouse 488 lot no. 898230 and Alexa Anti-Rabbit 568 lot no. 757102, Invitrogen) diluted 1:1000. It should be noted that the $\mathrm{TH}$ antibody used only detects TH1 in the zebrafish (Chen et al., 2009).

\section{MICROSCOPY AND IMAGING}

In situ hybridization samples were observed under a Leica DM IRB inverted microscope with an attached Leica DFC490 camera. Multifocus images were taken using Leica application suite version 2.7.0 software (Leica Microsystems CMS GmbH, Switzerland).

Immunofluorescent samples were visualized using a Leica SP2 AOBS confocal microscope (Leica Microsystems GmbH, Mannheim, Germany). The images were acquired using a HC PL APO $20 \times / 0.70 \mathrm{CS}$ objective. For detection of the fluorophores, a $488 \mathrm{~nm}$ argon laser and a $568 \mathrm{~nm}$ diode laser were used. The emission was collected at $500-550 \mathrm{~nm}$ for the $488 \mathrm{~nm}$ laser and at $600-700 \mathrm{~nm}$ for the $568 \mathrm{~nm}$ laser. The distance between stack planes was set at approximately $1 \mu \mathrm{m}$. The image stacks were imported to Fiji [open source imaging software (Schindelin et al., 2012)] for cell number quantification. Statistical analysis of cell numbers was done using One-Way ANOVA.

\section{NEUROTRANSMITTER MEASUREMENT BY HIGH-PERFORMANCE LIOUID CHROMATOGRAPHY}

For analysis of catecholamine levels 15-20 whole larvae were sonicated in 10 volumes of $2 \%$ perchloric acid, centrifuged for $30 \mathrm{~min}$ at $15,000 \mathrm{~g}$ after which $10 \mu \mathrm{L}$ of filtered supernatant was injected into high-performance liquid chromatography (HPLC) system equipped with a Waters Concorde electrochemical detector set to a potential $+0.80 \mathrm{~V}$, column oven and a column Gemini C18 $5 \mu \mathrm{m} 150 \times 4.60 \mathrm{~mm}$ (Phenomenex, Torrance, CA, USA). The mobile phase consisted of purified water with $8 \%$ methanol, $50 \mathrm{mM}$ citric acid, $1.5 \mathrm{mM}$ 1-octanesulfonic acid, $0.05 \mathrm{mM}$ EDTA, and $50 \mathrm{mM}$ phosphoric acid. The column temperature was set at $37^{\circ} \mathrm{C}$ and the flow rate at $1 \mathrm{ml} / \mathrm{min}$. System control, data acquisition and analysis were performed using Waters Empower software (Waters, Milford, MA). Concentrations of the catecholamines and metabolites were calculated from standard curves which were linear from $10 \mathrm{nM}$ to $1 \mu \mathrm{M}$. The protocol for histamine measurement has been described earlier (Eriksson et al., 1998). In order to normalize the data, sample protein concentration was measured using the Pierce ${ }^{\odot}$ BCA Protein Assay Kit (Thermo Fisher Scientific INC., Rockford, IL, USA). The measured concentration was normalized per protein, and the data is reported as percent of the average of the control group. Statistical analysis was done using One-Way ANOVA followed by Tukey's post hoc test.

\section{RESULTS}

\section{THE EFFECT OF ETHANOL ON SWIMMING BEHAVIOR}

We measured the total swimming distance and the mean values for meander, turn angle and angular velocity during the 10-min treatment period (Figure 1). The 1.50\% ethanol concentration increased the total swimming distance significantly $(p<0.05)$. A $3.00 \%$ concentration did not have any effect on the total distance moved. We also observed that the group treated with $1.50 \%$ ethanol showed a significant increase in mean angular velocity $(p<0.001)$, while the other treatment concentrations did not have any effect on this parameter. Mean meander and turn angle remained unchanged across all groups, except for a significant yet small change in turn angle observed between the 1.50 and $3.00 \%$ groups that was inconsistently observed between trials. We therefore do not find it to be of any relevance (Figures 1B,D).

In order to further understand the effect on the total distance moved, we analysed the parameter in 1-min intervals (Figure 1E). This revealed that the $3.00 \%$ concentration actually had a strong stimulatory effect on locomotion during the first few minutes of the treatment, but this effect subsided quickly and locomotor activity clearly diminished in the group for the remaining duration of the trial [time effect, $F_{(9,1791)}=75.20, p<0.0001$ ]. This sedative effect explained why there was no apparent effect on the total distance moved throughout the trial. This sedative effect was not observed for the 0.75 and $1.50 \%$ groups. The magnitude of the initial increase in locomotor activity appeared 


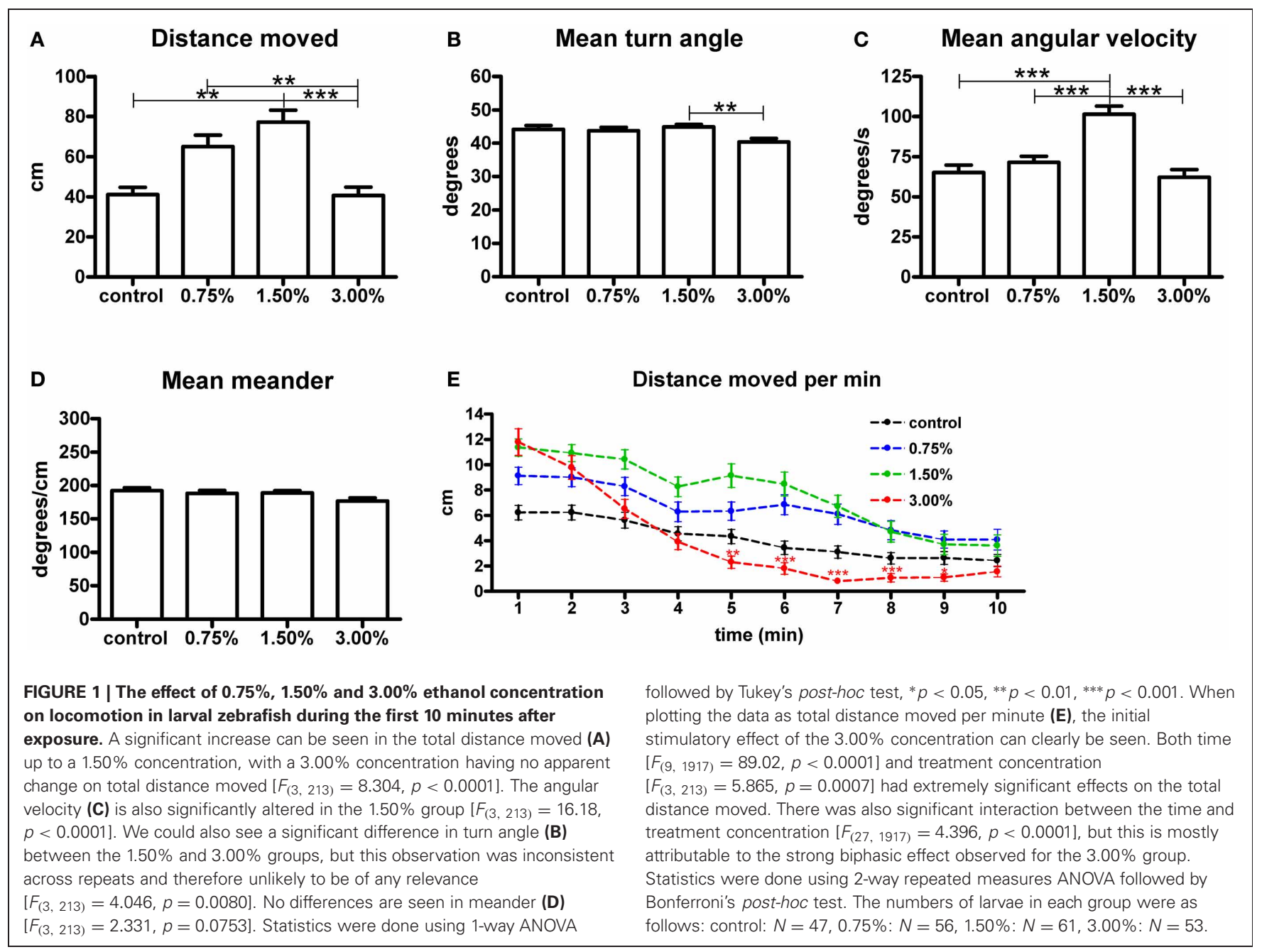

to be dose-dependent [treatment effect, $F_{(3,1791)}=8.21$, $p<0.0001]$.

\section{CHANGES IN TYROSINE HYDROXYLASE 1, TYROSINE HYDROXYLASE 2,} AND HISTIDINE DECARBOXYLASE TRANSCRIPT LEVELS

The amount of mRNA for each of the three rate-limiting enzymes in the biosynthesis of dopamine and histamine showed a clear dose-dependent increase trend following exposure to ethanol (Figure 2). The zebrafish has two tyrosine hydroxylase isoforms, TH1 and TH2 (Chen et al., 2009). Both th1 and th2 mRNA levels were significantly increased by the $3.00 \%$ ethanol treatment ( $p<0.05$ for th $1, p<0.01$ for th 2$)$. The increase in $h d c$ mRNA was also significant $(\mathrm{p}<0.01)$. These results indicate that ethanol directly or indirectly activates synthesis of the enzymes regulating the synthesis of dopamine and histamine in zebrafish almost immediately following exposure.

\section{IMMUNOHISTOCHEMISTRY AND in situ HYBRIDIZATION}

We observed no change in the expression pattern of th1, th2, and $h d c$ by in situ hybridization in any of the groups after a 10-min treatment (data not shown). These results were supported by immunohistochemistry, which also showed no changes in distribution patterns of $\mathrm{TH} 1$ - and histamine-immunoreactive cells and fibers (Figure 3, data not shown for TH1). This was verified by counting the number of histamine- and $\mathrm{TH}$ immunoreactive cells in the hypothalamus and preoptic group [groups 3 and 4 as defined by Sallinen et al. (2009b)], respectively. No change in cell numbers was seen ( $p>0.05$, Figure 4).

In order to determine if a prolonged treatment would alter the morphology of the histaminergic and dopaminergic systems, we repeated the in situ hybridization using a 30-min ethanol treatment. For this experiment, only the $1.50 \%$ ethanol dose was used, based on the acquired behavioral data. The longer treatment period did not show any difference in the expression patterns of $h d c$, th 1 , and th 2 between the $1.50 \%$ group and the control (Figure 5, data not shown for $h d c$ ). Immunohistochemistry also showed that the number and distribution of TH1immunoreactive cells remained unchanged in the preoptic group after the extended ethanol treatment $(p>0.05$, Figures 4, 6).

CHANGES IN DOPAMINE AND HISTAMINE LEVELS OBSERVED BY HPLC The higher ethanol concentrations resulted in significantly decreased dopamine levels after a 10 -min treatment $(p<0.05$, 

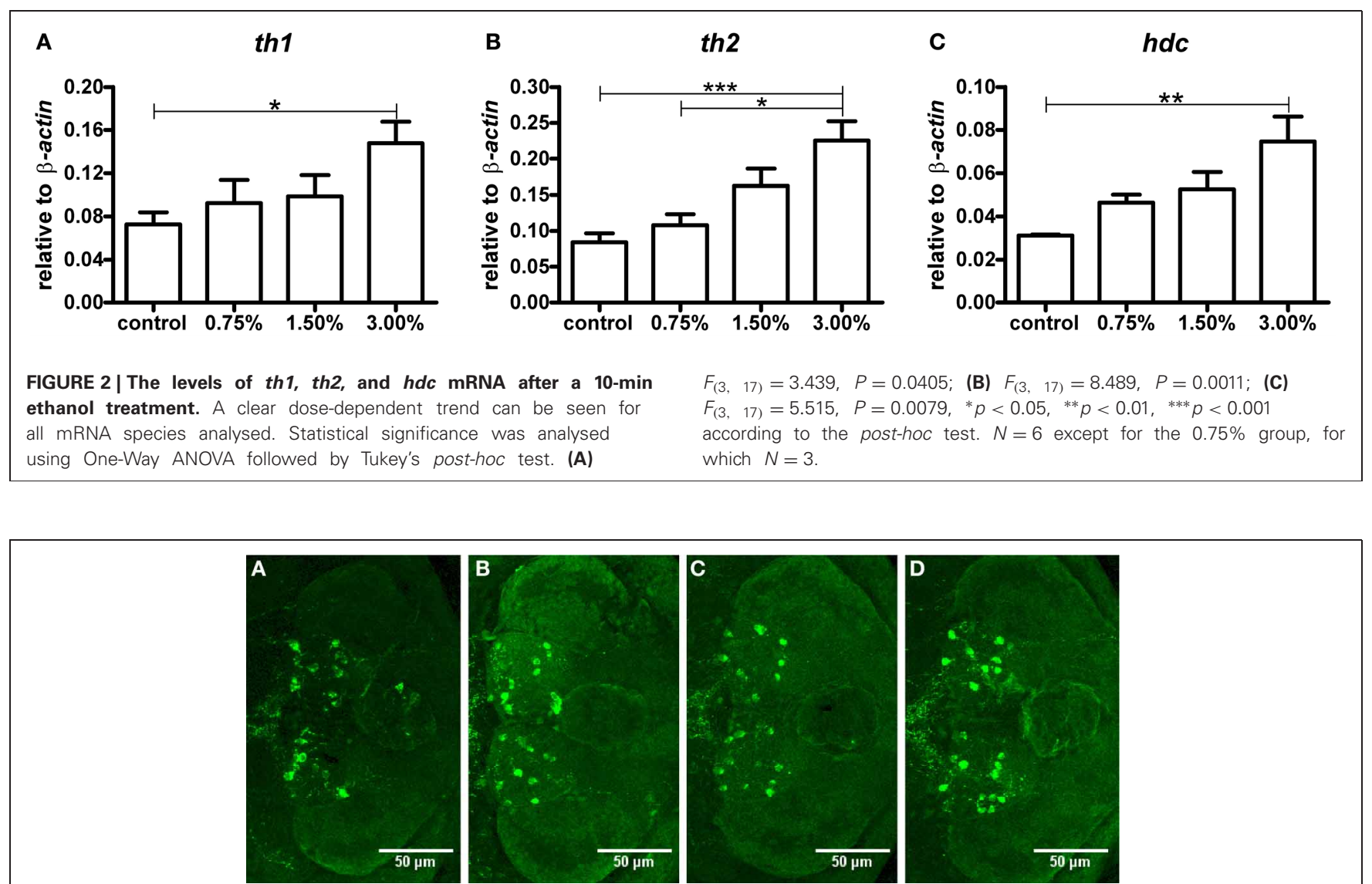

FIGURE 3 | Distribution of histamine-immunoreactive cells after a 10-min ethanol treatment. (A) Control, (B) $0.75 \%$ ethanol, (C) $1.50 \%$ ethanol, (D) $3.00 \%$ ethanol. No changes are seen between the groups, which are further confirmed by cell counting statistics in Figure 4. $N=13-15$ larvae per treatment group.
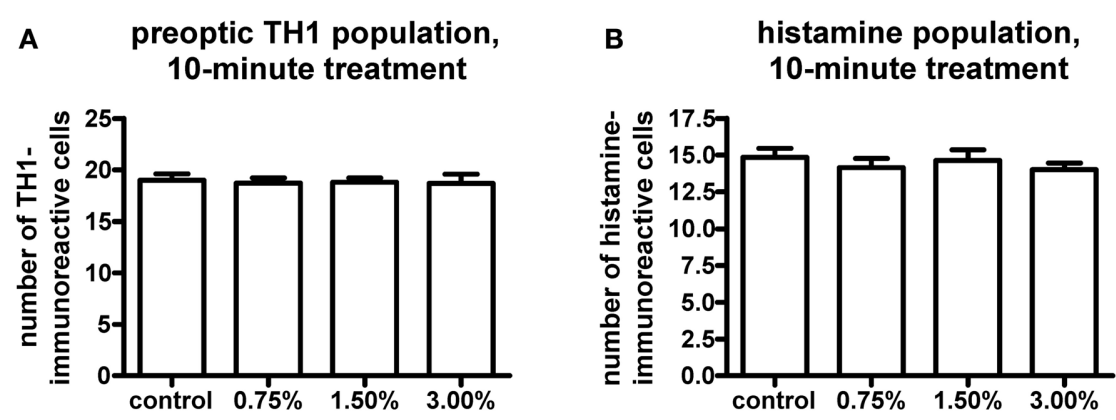

\section{C preoptic TH1 population, 30-minute treatment}

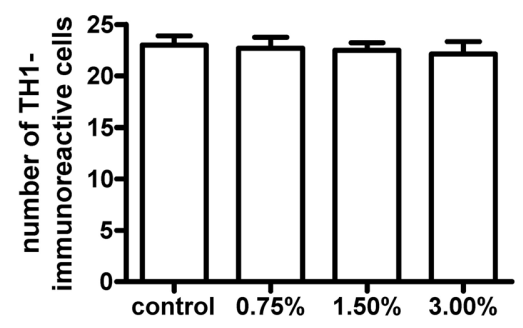

FIGURE 4 | Cell numbers in the histaminergic and preoptic dopaminergic populations after the ethanol treatment. A 10-min ethanol treatment had no effect on either population. In order to see if any change was occurring after a longer treatment, the preoptic dopaminergic population was further analysed after a 30-min treatment.
The longer treatment also did not show any changes in cell numbers. Statistical analysis was done using One-Way ANOVA. (A) $F_{(3,52)}=0.05260, p=0.9839 ;$ (B) $F_{(3,52)}=0.4550, p=0.7149$; (C) $F_{(3,33)}=0.1273, p=0.9432 . N=13-15$ for the $10-\mathrm{min}$ treatment, $N=8-10$ for the 30-min treatment.
Figure 7A). Curiously, the $0.75 \%$ group did not show a decrease in dopamine. None of the dopamine metabolites analysed [3,4dihydroxyphenylacetic acid (DOPAC) and homovanillic acid (HVA)] showed any significant changes (Figures 7B,C). For the metabolic pathways of dopamine, please refer to Figure 8. HVA levels showed a slight dose-dependent decreasing trend, but this observation did not reach statistical significance $(p>0.05)$. The levels of other metabolites remained consistent across all groups. In order to see if the upregulation of th 1 and th 2 observed after a 10-min treatment would counteract the decrease in dopamine observed, we measured the dopamine levels again after a 30-min treatment (Figure 7F). At this point, the difference in dopamine 
levels observed between the groups was smaller and statistically not significant, indicating that the dopamine loss might indeed be compensated.

Out of other neurotransmitters, histamine, and noradrenaline were measured. Noradrenaline levels were unaffected by the treatment (Figure 7D). Only a non-significant decreasing trend in histamine levels $(p>0.05)$ was seen (Figure 7E).

\section{DISCUSSION}

Our results showed that ethanol concentrations of 0.75 and $1.50 \%$ have an acute stimulatory effect on locomotion in larval zebrafish, while a $3.00 \%$ concentration resulted in a brief increase in activity, quickly followed by a strong decrease of locomotion. This corresponds well with results obtained from
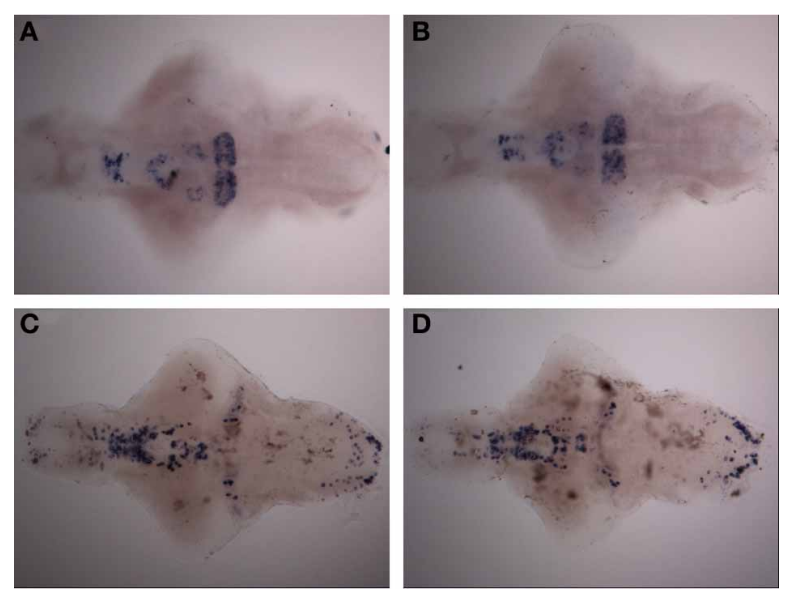

FIGURE 5 | Expression pattern of th1 and th2 after a 30-min ethanol treatment. (A) th2 expression pattern after 30 -min of $1.50 \%$ ethanol. (B) th2 expression in the control group. (C) th1 expression pattern after 30-min of $1.50 \%$ ethanol. (D) th 1 expression in the control group. No differences can be seen between the groups. $N=10$ larvae per treatment group. earlier studies (Lockwood et al., 2004; Macphail et al., 2009; De Esch et al., 2012), and shows that the fish strain used here displays a concentration-dependent increase in locomotor activity following exposure to ethanol. Out of these earlier studies, only Lockwood et al. (2004) have analysed the movement activity immediately after administration of ethanol, showing a large difference in the magnitude of the ethanol-induced stimulation between the $\mathrm{AB}$ and WIK wild-type strains of zebrafish (Lockwood et al., 2004). Our Turku strain reacted similar to the WIK strain, showing a rather modest increase in locomotor activity. The initial stimulatory effect we observed for the $3.00 \%$ treatment could be explained by a similar biphasic effect that was observed in a study with mice (Crabbe et al., 1982), where ethanol doses cause a similar effect for $\mathrm{C} 57 \mathrm{BL} / 6 \mathrm{~N}$ mice. Another possibility is that the initial response to ethanol would be caused by the presence of an irritating substance. This was, however, also investigated by Lockwood et al. (2004), who showed that a $1.50 \%$ methanol treatment had no effect on locomotion (Lockwood et al., 2004). We therefore find it reasonable to interpret the observed stimulation as an effect attributable to the pharmacological properties of ethanol and not as an unspecific reaction to a noxious substance. Our data also suggests that ethanol changes the swimming pattern of larval zebrafish, as shown by the increase in mean angular velocity for the $1.50 \%$ group. This might indicate an impairment of motor coordination after a $1.50 \%$ ethanol treatment, which is a well-known effect of ethanol (Carta et al., 2004).

The main focus of our study was to characterize the dynamic changes in in key neurotransmitters associated with locomotor activation following ethanol exposure. The stimulatory effect of ethanol is known to be linked to the activation of mesolimbic dopaminergic pathways in mice (Meyer et al., 2009). TH is the rate-limiting enzyme of dopamine synthesis. Zebrafish have two isoforms of $\mathrm{TH}$ called $\mathrm{TH} 1$ and $\mathrm{TH} 2$. The distribution of th1 and th2 cells in the zebrafish brain show a complementary pattern, with th1 being the dominant isoform in the brain (Chen
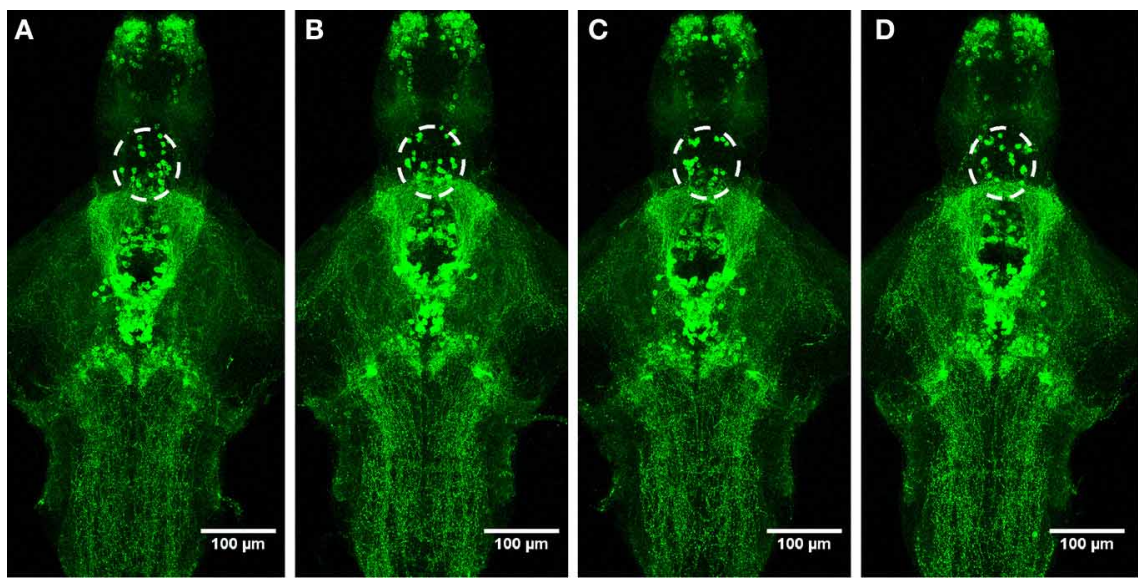

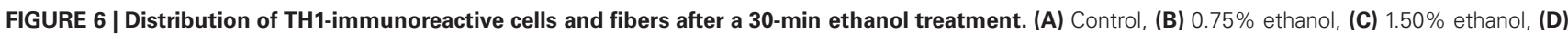
$3.00 \%$ ethanol. No changes are seen between the groups. $N=8-10$ larvae per treatment group. 


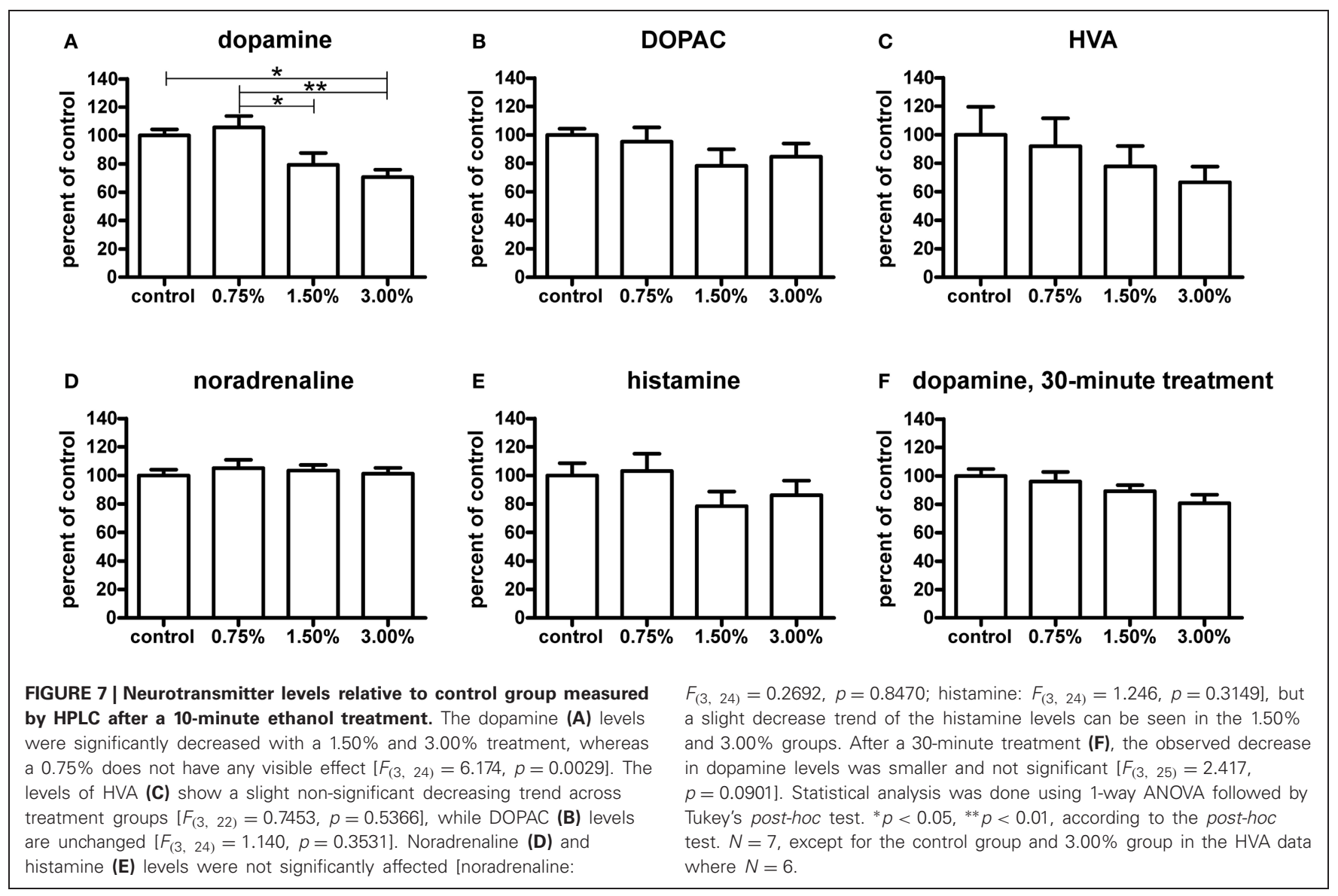

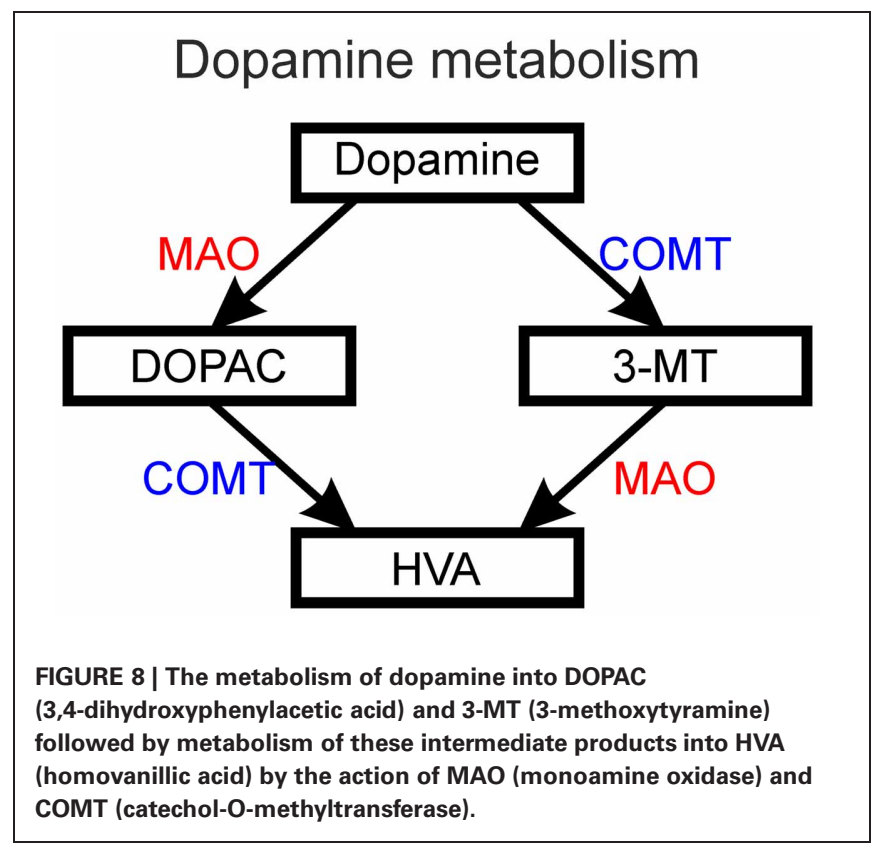

et al., 2009). Since an increase in th2 has been shown to be affected by different states of behavior (Pavlidis et al., 2011), we hypothesized that ethanol might affect either enzyme only. This was, however, not the case, as we saw an increase in transcript numbers of both th genes. It would therefore seem that ethanol has a general stimulatory effect on dopamine synthesis in the zebrafish, since these $\mathrm{TH}$ forms are expressed in different neuron populations with different projections (Chen et al., 2009). This is also supported by our data obtained by in situ hybridization, which showed that the patterns of th 1 and th 2 expression in the brain remained unchanged, and immunohistochemistry, which showed that the number and pattern of $\mathrm{TH} 1$-immunoreactive cells remained unaffected.

As different populations of dopaminergic neurons project widely to key areas involved in motor regulation, for instance the subpallium (Rink and Wullimann, 2001; Tay et al., 2011), the nucleus of the medial longitudinal fascicle (McLean and Fetcho, 2004) and even the spinal motor neurons themselves (McLean and Fetcho, 2004), it is of key interest to know if the stimulatory effect of ethanol is mediated by one or several of these systems, or through a diffuse activation of the dopaminergic network. In mammalian models, the activation of locomotion has been strongly linked to effects on the VTA dopaminergic neurons, demonstrating, for instance, increased activation of VTA neurons (Gessa et al., 1985) increased th mRNA levels in the VTA (Oliva et al., 2008) and increased dopamine release in VTA target areas (Di Chiara and Imperato, 1986). We were unable to measure the release of dopamine in different brain areas due to the small size of the zebrafish brain, but our data obtained by qPCR, immunohistochemistry and in situ hybridization supports the 
theory of a diffuse activation of the dopaminergic network, as discussed in the previous section. It should, however, be noted that our analysis on the role of $\mathrm{TH} 2$ in this process is incomplete, as we did not have access to a working characterized $\mathrm{TH} 2$ antibody during this study. Also, although the activation of the dopaminergic system might be general, this does not directly imply that all possible pathways participating in the stimulation of locomotion are activated. Our results give reason for future studies further investigating this effect. In situ hybridization and immunocytochemistry showed that there was no change in cell numbers expressing the th transcripts following ethanol. The activation thus occurred in cells which also under control conditions express th1 or th2.

The higher ethanol concentrations seemed to have a decreasing effect on dopamine levels in the zebrafish after a 10-min treatment. The increase in th 1 and th 2 transcription might be a part of a mechanism to increase synthesis of $\mathrm{TH}$ and therefore the synthesis of dopamine, which is supported by our data showing no significant differences between treatment groups after a 30-min treatment. A decrease in dopamine also implies that ethanol is stimulating dopamine release, which is a well-known phenomenon in rodents (Di Chiara and Imperato, 1986, 1988). The increase in dopamine release would explain the stimulating effect on locomotion. We were, however, unable to directly demonstrate an increase in dopamine release, as microdialysis is not feasible in larval zebrafish. Although earlier studies with zebrafish have demonstrated an increase in dopamine following an acute ethanol dose (Chatterjee and Gerlai, 2009; Gerlai et al., 2009), these results were obtained using adult zebrafish and lower ethanol treatment doses. Since the pharmacokinetic differences between larval and adult zebrafish have yet to be elucidated, it is difficult to compare our results and results obtained in adult fish. In our study, only the 1.50 and $3.00 \%$ ethanol concentrations resulted in a decrease in dopamine levels, while a $0.75 \%$ concentration had no significant effect. It is therefore entirely possible that only higher ethanol doses cause dopamine levels to actually decrease, while the increased synthesis implicated by increased th 1 and th 2 transcription compensates the decrease at lower doses. Curiously, we did not see an increase of any of the dopamine metabolites, which would be expected if dopamine levels were decreased due to increased release and metabolism. Instead, we saw that HVA levels actually decreased slightly in a dose-dependent manner. The kinetics of the metabolism of catecholamines in zebrafish is not known, and more research will be necessary in order to explain this change. One might argue that the decrease in dopamine would indicate some kind of acute toxic effect on dopaminergic neurons. We find this to be unlikely, however, as we could not see any change in the expression pattern of th1 and th 2 or TH1immunoreactive neurons even after a longer, 30-min ethanol treatment.

Although ethanol treatments of similar concentration have proven to be teratogenic in younger zebrafish (Reimers et al., 2004; Arenzana et al., 2006), there is evidence of changes in the expression of alcohol dehydrogenase 3 ( $\mathrm{ADH} 3)$ during fish development, which implicates possible age-dependent differ- ences in the metabolism and toxicity of ethanol on zebrafish (Dasmahapatra et al., 2001). Internal ethanol concentrations in the $0.75-3.00 \%$ range are likely to be lethal in mammals. It was shown by Lockwood et al. that a 20-min treatment of 7-dayold larval zebrafish resulted in internal ethanol concentrations of $25 \mathrm{mM}(0.12 \%)$ and $71 \mathrm{mM}(0.33 \%)$ after treatment with 1.5 and 3.0\% ethanol solutions, respectively (Lockwood et al., 2004). Internal concentrations around this range have been reported in studies with mice (Nuutinen et al., 2011), and the use of similar ethanol concentrations in other studies with zebrafish (Lockwood et al., 2004; Macphail et al., 2009) further justifies the use of high ethanol concentrations in the treatment solutions.

A novel discovery in our study was that ethanol increased the transcription of HDC, the rate-limiting enzyme of histamine synthesis. Recent studies in mice have demonstrated that histamine indeed is essential in ethanol-induced locomotor activation and also in the associated reward-mechanism (Nuutinen et al., 2010, 2011). Again, the morphology and number of the histaminergic system was unaffected, as verified by in situ hybridization and immunohistochemistry, which would support the assumption that the increase is due to an upregulation of the $h d c$ gene in cells which express hdc under normal conditions. In zebrafish, the histaminergic neurons show life-long plasticity and there numbers are regulated by presenilin 1 through Notch1 (Sundvik et al., 2013). They are essential in regulation of e.g., dark flash swimming response (Sundvik et al., 2011) and histamine regulates vigilance or anxiety-like behaviors (Peitsaro et al., 2003). Also in rodents activity of histaminergic neurons is linked to increased vigilance and cognitive capacity (Haas and Panula, 2003; Anaclet et al., 2009). Further studies are necessary in order to elucidate the purpose and effects of the increase in $h d c$.

In conclusion, this study shows evidence that an acute ethanol treatment increases locomotor activity with moderate doses of ethanol in larval zebrafish. At high concentration, an acute increase in locomotor activity is associated with a decline in dopamine levels and induction of th1, th2, and $h d c$ without any change in the anatomy of the dopaminergic and histaminergic systems, indicating that ethanol might have a diffuse stimulatory effect on these systems in zebrafish. In addition, this study provides evidence that a $1.50 \%$ ethanol concentration causes impairment of motor control in larval zebrafish, as seen by the increase in fish angular velocity. The decline in dopamine levels after a 10-min treatment might result from strongly increased dopamine release followed by upregulation of both th forms, resulting in partially normalized dopamine levels after a 30 -min treatment. This provides a basis for further study, in order to fully understand the effect of ethanol on the dopaminergic and histaminergic systems in zebrafish.

\section{ACKNOWLEDGMENTS}

We thank Henri Koivula, B.Sc., and Susanna Norrbacka, B.Sc., for fish management and expert technical assistance. Supported by the Academy of Finland and Sigrid Juselius Foundation. 


\section{REFERENCES}

Anaclet, C., Parmentier, R., Ouk, K., Guidon, G., Buda, C., Sastre, J. P., et al. (2009). Orexin/hypocretin and histamine: distinct roles in the control of wakefulness demonstrated using knock-out mouse models. J. Neurosci. 29, 14423-14438. doi: 10.1523/JNEUROSCI.2604-09.2009

Arenzana, F. J., Carvan, M. J. 3rd, Aijon, J., Sanchez-Gonzalez, R., Arevalo, R., and Porteros, A. (2006). Teratogenic effects of ethanol exposure on zebrafish visual system development. Neurotoxicol. Teratol. 28, 342-348. doi: 10.1016/j.ntt.2006.02.001

Carta, M., Mameli, M., and Valenzuela, C. F. (2004). Alcohol enhances GABAergic transmission to cerebellar granule cells via an increase in Golgi cell excitability. J. Neurosci. 24, 3746-3751. doi: 10.1523/JNEUROSCI.0067-04.2004

Chatterjee, D., and Gerlai, R. (2009). High precision liquid chromatography analysis of dopaminergic and serotoninergic responses to acute alcohol exposure in zebrafish. Behav. Brain Res. 200, 208-213. doi: 10.1016/j.bbr.2009.01.016

Chen, Y. C., Priyadarshini, M., and Panula, P. (2009). Complementary developmental expression of the two tyrosine hydroxylase transcripts in zebrafish. Histochem. Cell Biol. 132, 375-381. doi: 10.1007/s00418009-0619-8

Cohen, C., Perrault, G., and Sanger, D. J. (1997). Evidence for the involvement of dopamine receptors in ethanol-induced hyperactivity in mice. Neuropharmacology 36, 1099-1108. doi: 10.1016/s00283908(97)00100-7

Crabbe, J. C. Jr., Johnson, N. A., Gray, D. K., Kosobud, A., and Young, E. R. (1982). Biphasic effects of ethanol on open-field activity: sensitivity and tolerance in C57BL/6N and $\mathrm{DBA} / 2 \mathrm{~N}$ mice. J. Comp. Physiol. Psychol. 96, 440-451. doi: 10.1037/h0077898

Dasmahapatra, A. K., Doucet, H. L., Bhattacharyya, C., and Carvan, M. J. 3rd. (2001). Developmental expression of alcohol dehydrogenase $(\mathrm{ADH} 3)$ in zebrafish (Danio rerio). Biochem. Biophys. Res. Commun. 286, 1082-1086. doi: 10.1006/bbrc. 2001.5511

De Esch, C., Van Der Linde, H., Slieker, R., Willemsen, R., Wolterbeek, A., Woutersen, R., et al. (2012). Locomotor activity assay in zebrafish larvae: influence of age, strain and ethanol. Neurotoxicol. Teratol. 34, 425-433. doi: 10.1016/j.ntt.2012.03.002
Di Chiara, G., and Imperato, A. (1986). Preferential stimulation of dopamine release in the nucleus accumbens by opiates, alcohol, and barbiturates: studies with transcerebral dialysis in freely moving rats. Ann. N.Y. Acad. Sci. 473, 367-381. doi: 10.1111/j.17496632.1986.tb23629.x

Di Chiara, G., and Imperato, A. (1988). Drugs abused by humans preferentially increase synaptic dopamine concentrations in the mesolimbic system of freely moving rats. Proc. Natl. Acad. Sci. U.S.A. 85, 5274-5278. doi: 10.1073/pnas.85.14.5274

Eriksson, K. S., Peitsaro, N., Karlstedt, K., Kaslin, J., and Panula, P. (1998). Development of the histaminergic neurons and expression of histidine decarboxylase mRNA in the zebrafish brain in the absence of all peripheral histaminergic systems. Eur. J. Neurosci. 10, 3799-3812. doi: 10.1046/j.1460-9568.1998.00394.x

Farrar, A. M., Segovia, K. N., Randall, P. A., Nunes, E. J., Collins, L. E., Stopper, C. M., et al. (2010). Nucleus accumbens and effortrelated functions: behavioral and neural markers of the interactions between adenosine A2A and dopamine D2 receptors. Neuroscience 166, 1056-1067. doi: 10.1016/j.neuroscience.2009.12.056

Gerlai, R., Chatterjee, D., Pereira, T., Sawashima, T., and Krishnannair, R. (2009). Acute and chronic alcohol dose: population differences in behavior and neurochemistry of zebrafish. Genes Brain Behav. 8, 586-599. doi: 10.1111/j.1601183X.2009.00488.x

Gerlai, R., Lahav, M., Guo, S., and Rosenthal, A. (2000). Drinks like a fish: zebra fish (Danio rerio) as a behavior genetic model to study alcohol effects. Pharmacol. Biochem. Behav. 67, 773-782. doi: 10.1016/S0091-3057(00)00422-6

Gessa, G. L., Muntoni, F., Collu, M., Vargiu, L., and Mereu, G. (1985). Low doses of ethanol activate dopaminergic neurons in the ventral tegmental area. Brain Res. 348, 201-203. doi: 10.1016/00068993(85)90381-6

Haas, H., and Panula, P. (2003). The role of histamine and the tuberomamillary nucleus in the nervous system. Nat. Rev. Neurosci. 4, 121-130. doi: 10.1038/nrn1034

Irons, T. D., Macphail, R. C., Hunter, D. L., and Padilla, S. (2010). Acute neuroactive drug exposures alter locomotor activity in larval zebrafish. Neurotoxicol. Teratol. 32, 84-90. doi: 10.1016/j.ntt.2009.04.066
Kashem, M. A., Ahmed, S., Sarker, R., Ahmed, E. U., Hargreaves, G. A., and McGregor, I. S. (2012). Longterm daily access to alcohol alters dopamine-related synthesis and signaling proteins in the rat striatum. Neurochem. Int. 61, 1280-1288. doi: 10.1016/j.neuint.2012.08.013

Kaslin, J., and Panula, P. (2001). Comparative anatomy of the histaminergic and other aminergic systems in zebrafish (Danio rerio). J. Comp. Neurol. 440, 342-377. doi: 10.1002/cne. 1390

Kelly, P. H., Seviour, P. W., and Iversen, S. D. (1975). Amphetamine and apomorphine responses in the rat following 6-OHDA lesions of the nucleus accumbens septi and corpus striatum. Brain Res. 94, 507-522. doi: 10.1016/0006-8993 (75)90233-4

Kily, L. J., Cowe, Y. C., Hussain, O., Patel, S., McElwaine, S., Cotter, F. E., et al. (2008). Gene expression changes in a zebrafish model of drug dependency suggest conservation of neuro-adaptation pathways. J. Exp. Biol. 211, 1623-1634. doi: 10.1242/jeb.014399

Lee, Y. K., Park, S. W., Kim, Y. K., Kim, D. J., Jeong, J., Myrick, H., et al. (2005). Effects of naltrexone on the ethanol-induced changes in the rat central dopaminergic system. Alcohol Alcohol. 40, 297-301. doi: 10.1093/alcalc/agh163

Lintunen, M., Raatesalmi, K., Sallmen, T., Anichtchik, O., Karlstedt, K., Kaslin, J., et al. (2002). Low brain histamine content affects ethanol-induced motor impairment. Neurobiol. Dis. 9, 94-105. doi: 10.1006/nbdi.2001.0453

Livak, K. J., and Schmittgen, T. D. (2001). Analysis of relative gene expression data using real-time quantitative $\mathrm{PCR}$ and the 2(-Delta Delta $\mathrm{C}(\mathrm{T})$ ) Method. Methods 25, 402-408. doi: 10.1006/meth.2001.1262

Lockwood, B., Bjerke, S., Kobayashi, K., and Guo, S. (2004). Acute effects of alcohol on larval zebrafish: a genetic system for large-scale screening. Pharmacol. Biochem. Behav. 77, 647-654. doi: 10.1016/j.pbb.2004.01.003

Macphail, R. C., Brooks, J., Hunter, D. L., Padnos, B., Irons, T. D., and Padilla, S. (2009). Locomotion in larval zebrafish: influence of time of day, lighting and ethanol. Neurotoxicology 30, 52-58. doi: 10.1016/j.neuro.2008.09.011

Mathur, P., Berberoglu, M. A., and Guo, S. (2011). Preference for ethanol in zebrafish following a single exposure. Behav.
Brain Res. 217, 128-133. doi: 10.1016/j.bbr.2010.10.015

McLean, D. L., and Fetcho, J. R. (2004) Relationship of tyrosine hydroxylase and serotonin immunoreactivity to sensorimotor circuitry in larval zebrafish. J. Comp. Neurol. 480 , 57-71. doi: 10.1002/cne.20281

Meyer, P. J., Meshul, C. K., and Phillips, T. J. (2009). Ethanol- and cocaineinduced locomotion are genetically related to increases in accumbal dopamine. Genes Brain Behav. 8, 346-355. doi: 10.1111/j.1601183X.2009.00481.x

Mogenson, G. J., Jones, D. L., and Yim, C. Y. (1980). From motivation to action: functional interface between the limbic system and the motor system. Prog. Neurobiol. 14, 69-97. doi: 10.1016/0301-0082(80)90018-0

Navarrete, F., Rubio, G., and Manzanares, J. (2013). Effects of naltrexone plus topiramate on ethanol self-administration and tyrosine hydroxylase gene expression changes. Addict. Biol. doi: 10.1111/adb.12058. [Epub ahead of print].

Nuutinen, S., Karlstedt, K., AittaAho, T., Korpi, E. R., and Panula, P. (2010). Histamine and H3 receptor-dependent mechanisms regulate ethanol stimulation and conditioned place preference in mice. Psychopharmacology 208, 75-86. doi: 10.1007/s00213-0091710-5

Nuutinen, S., Lintunen, M., Vanhanen, J., Ojala, T., Rozov, S., and Panula, P. (2011). Evidence for the role of histamine $\mathrm{H} 3$ receptor in alcohol consumption and alcohol reward in mice. Neuropsychopharmacology 36, 2030-2040. doi: 10.1038/ npp. 2011.90

Oliva, J. M., Ortiz, S., Perez-Rial, S., and Manzanares, J. (2008). Time dependent alterations on tyrosine hydroxylase, opioid and cannabinoid CB1 receptor gene expressions after acute ethanol administration in the rat brain. Eur. Neuropsychopharmacol. 18, 373-382. doi: 10.1016/j.euro neuro.2007.09.001

Panula, P., Airaksinen, M. S., Pirvola, U., and Kotilainen, E. (1990). A histamine-containing neuronal system in human brain. Neuroscience 34, 127-132. doi: 10.1016/03064522(90)90307-P

Papanicolaou, J., and Fennessy, M. R. (1980). The acute effect of ethanol on behaviour, body temperature, and brain histamine in mice. Psychopharmacology 72, 73-77. doi: 10.1007/BF00433809

Pavlidis, M., Sundvik, M., Chen, Y. C., and Panula, P. (2011). 
Adaptive changes in zebrafish brain in dominant-subordinate behavioral context. Behav. Brain Res. 225, 529-537. doi: 10.1016/ j.bbr.2011.08.022

Peitsaro, N., Kaslin, J., Anichtchik, O. V., and Panula, P. (2003). Modulation of the histaminergic system and behaviour by alpha-fluoromethylhistidine in zebrafish. J. Neurochem. 86, 432-441. doi: 10.1046/j.1471-4159. 2003.01850.x

Peitsaro, N., Sundvik, M., Anichtchik, O. V., Kaslin, J., and Panula, P. (2007). Identification of zebrafish histamine $\mathrm{H} 1, \mathrm{H} 2$ and $\mathrm{H} 3$ receptors and effects of histaminergic ligands on behavior. Biochem. Pharmacol. 73, 1205-1214. doi: 10.1016/j.bcp.2007.01.014

Rawat, A. K. (1980). Development of histaminergic pathways in brain as influenced by maternal alcoholism. Res. Commun. Chem. Pathol. Pharmacol. 27, 91-103.

Reimers, M. J., Flockton, A. R., and Tanguay, R. L. (2004). Ethanol- and acetaldehydemediated developmental toxicity in zebrafish. Neurotoxicol. Teratol. 26, 769-781. doi: 10.1016/j.ntt.2004. 06.012

Rink, E., and Wullimann, M. F. (2001). The teleostean (zebrafish) dopaminergic system ascending to the subpallium (striatum) is located in the basal diencephalon (posterior tuberculum).
Brain Res. 889, 316-330. doi: 10.1016/S0006-8993(00)03174-7

Sallinen, V., Kolehmainen, J., Priyadarshini, M., Toleikyte, G., Chen, Y. C., and Panula, P. (2010). Dopaminergic cell damage and vulnerability to MPTP in Pink1 knockdown zebrafish. Neurobiol. Dis. 40, 93-101. doi: 10.1016/j.nbd.2010.06.001

Sallinen, V., Sundvik, M., Reenila, I., Peitsaro, N., Khrustalyov, D., Anichtchik, O., et al. (2009a). Hyperserotonergic phenotype after monoamine oxidase inhibition in larval zebrafish. J. Neurochem. 109, 403-415. doi: 10.1111/j.1471-4159.2009.05986.x

Sallinen, V., Torkko, V., Sundvik, M., Reenila, I., Khrustalyov, D., Kaslin, J., et al. (2009b). MPTP and $\mathrm{MPP}+$ target specific aminergic cell populations in larval zebrafish. J. Neurochem. 108, 719-731. doi: 10.1111/j.1471-4159.2008.05793.x

Schindelin, J., Arganda-Carreras, I., Frise, E., Kaynig, V., Longair, M., Pietzsch, T., et al. (2012). Fiji: an open-source platform for biological-image analysis. Nat. Methods 9, 676-682. doi: 10.1038/ nmeth.2019

Subramanian, N., Mitznegg, P., and Estler, C. J. (1978). Ethanol-induced alterations in histamine content and release in the rat hypothalamus. Naunyn Schmiedebergs Arch. Pharmacol. 302, 119-121. doi: 10.1007/BF00586607
Sundvik, M., Chen, Y. C., and Panula, P. (2013). Presenilin1 regulates histamine neuron development and behavior in zebrafish, danio rerio. J. Neurosci. 33, 1589-1597. doi: 10.1523/JNEUROSCI.1802-12. 2013

Sundvik, M., Kudo, H., Toivonen, P., Rozov, S., Chen, Y. C., and Panula, P. (2011). The histaminergic system regulates wakefulness and orexin/hypocretin neuron development via histamine receptor $\mathrm{H} 1$ in zebrafish. FASEB J. 25, 4338-4347. doi: 10.1096/fj.11-188268

Tang, A., George, M. A., Randall, J. A., and Gonzales, R. A. (2003). Ethanol increases extracellular dopamine concentration in the ventral striatum in C57BL/6 mice. Alcohol Clin. Exp. Res. 27, 1083-1089. doi: 10.1097/01.ALC.0000075825. 14331.65

Tay, T. L., Ronneberger, O., Ryu, S., Nitschke, R., and Driever, W. (2011). Comprehensive catecholaminergic projectome analysis reveals single-neuron integration of zebrafish ascending and descending dopaminergic systems. Nat. Commun. 2, 171. doi: 10.1038/ncomms 1171

Thisse, C., and Thisse, B. (2008). High-resolution in situ hybridization to whole-mount zebrafish embryos. Nat. Protoc. 3, 59-69. doi: 10.1038/nprot.2007.514

Westerfield, M. (2000). "The zebrafish book," in A Guide for the Laboratory
Use of Zebrafish (Danio rerio). Eugene, OR: University of Oregon Press. Available online at: http://zfin.org/zf_info/zfbook/ zfbk.html

Yoshimoto, K., McBride, W. J., Lumeng, L., and Li, T. K. (1992). Alcohol stimulates the release of dopamine and serotonin in the nucleus accumbens. Alcohol 9, 17-22. doi 10.1016/0741-8329(92)90004-T

Conflict of Interest Statement: The authors declare that the research was conducted in the absence of any commercial or financial relationships that could be construed as a potential conflict of interest.

Received: 13 February 2013; accepted: 10 May 2013; published online: 31 May 2013.

Citation: Puttonen HAJ, Sundvik M, Rozov S, Chen Y-C and Panula P (2013) Acute ethanol treatment upregulates th1, th2, and hdc in larval zebrafish in stable networks. Front. Neural Circuits 7:102. doi: 10.3389/fncir.2013.00102

Copyright (0) 2013 Puttonen, Sundvik, Rozov, Chen, and Panula. This is an open-access article distributed under the terms of the Creative Commons Attribution License, which permits use, distribution and reproduction in other forums, provided the original authors and source are credited and subject to any copyright notices concerning any thirdparty graphics etc. 